\title{
Network Routing Optimization based on Mixed Particle Method Research
}

\author{
Sheng Yan Gang and Tong Fei \\ Qingdao Vocational and Technical College of Hotel Management \\ qd_syg@163.com,tongfei0181@163.com
}

\begin{abstract}
Particle swarm optimization (POS) algorithm is a reference to the mechanism of the biological predation community of a random search algorithm, the validity of its search, got extensive application in many ways.But the particle swarm algorithm has poor local search ability and easy early faults.So on the basis of it, combined with the genetic algorithm is proposed based on a mixed particle swarm algorithm.The new algorithm USES the similar to the mutation and crossover operation of genetic algorithm, speed and position update formula easier to express.To primary respectively after the particle group division evolution, global optimization to the formation of new species.Applied in the IP network quality of service routing optimization mixed POS algorithm, both meet the requirement of quality of service and will choose the path cost minimum as the optimization index.Through a series of simulation experiments of network routing, the improved algorithm has good effect.
\end{abstract}

Keywords: mixed particle method, network routing,optimize,

\section{Introduction}

The 21st century is the information society, people are enjoying the information society brings us endless convenience at the same time, also deeply felt the huge amounts of information to our challenge. Facing the challenge of huge amounts of information, there are two basic strategies, one is computing faster, in this respect, we see the representative to the information technology, the CPU in constant "evolution". The evolution of the CPU speed, however, seems to always miss information increase, the speed of the transmission. At present, the technology of the CPU into a position of "Moore's law" failure. Another basic strategy is to calculate the human to be more intelligent, is always the human nature in this respect the teacher and human being society system and physical system, biological system operation mechanism, establish and develop a research tool and means to resolve and overcome some difficulties in the research process. Typically have a genetic algorithm, artificial neural networks, evolutionary programming, ant colony optimization algorithm. These methods have been widely applied in engineering practice, there are many successful examples. And in the field of computational intelligence, there are two kinds of algorithm based on swarm intelligence, ant colony optimization algorithm and particle swarm optimization algorithm ${ }^{[1-3]}$. Before the simulation of the ant colony search food behavior, has been used successfully in many combinatorial optimization problems; The latter is the particle swarm optimization algorithm will be introduced in this paper.

Particle swarm optimization algorithm is invented by DrEberhart and DrKennedeya evolutionary computation technology, is a new branch in the field of evolutionary computation, it is the result of the study of birds and fish population movement behavior. Many social species, its relative social network helps to take care of the young individuals together, live together can share the food, and can provide the warning at the time of natural enemy invasion and collective defense, group of prey may be more effective than a single 
individual, when many pairs of eyes to find food at the same time, feeding efficiency can be improved. Due to the innovation and imitation and accumulation of cultural evolution, the build-up of solutions to problems, these solutions are based on group cooperation and communication. Culture to keep the standard, and try to meet or exceed the standard, like a ratchet can prevent sliding backwards, here it is. The ratchet effect.PSO model group biological behavior of social nature. Individual's interaction with each other at the same time, in learning their own experience and members of the species gradually moved into better area of the problem space.

Based on Darwin's "survival of the fittest, superior bad discard" evolutionary thought of genetic algorithm, particle swarm optimization algorithm is through collaboration between the individual to find the optimal solution. Refers to the biological sociologist E.O Wsilon a passage about biological group ", at least in theory, a member of a biological group can be from all the other members in this group from past experience accumulated in the process of searching for food and find benefit. So long as food source is information distributed in different places, this collaboration to bring advantage can become a decisive, among more than the disadvantages brought by the competition for food between individual "this passage mean information sharing makes evolutionary advantage in biology group, which is the basic idea of particle swarm optimization algorithm. Concept of particle swarm algorithm is simple, easy to implement, its only the short several lines of code, compared with other optimization algorithms, which is one of the advantages of it. Just a few years, PSO algorithm has been obtained great development, and has got good application in some field. Particle swarm algorithm has some classic optimization problems to solve and the practical application shows strong vitality and potential. It has with the traditional optimization algorithm (such as mathematical programming, dynamic programming, etc.) of different characteristics as follows:

1) It is a kind of method of uncertainty. Uncertainty reflects the physiological mechanism of nature, and is better than the deterministic algorithm in solving some specific problems.

2) It is not dependent on the optimization in the process of optimization problem of strict mathematical properties, such as continuity, conductivity and the objective function and constraint conditions of precise mathematical description.

3) It is a kind of intelligent algorithm based on multiple agents. Each agent through self-learning to improve their adaptability, through collaboration between each other at the same time to better adapt to the environment.

4) It has the potential of parallelism. The search process is not starting from a point, but at the same time from the multiple points simultaneously. This distributed multiple agent cooperation process is asynchronous concurrent, distributed parallel mode will greatly improve the operation efficiency and convergence speed of the algorithm.

Domestic and foreign research shows that the PSO is a powerful tool to solve the problem of complex system optimization. At first, PSO algorithm is mainly used for the optimization of continuous functions, was later applied to combinatorial optimization such as the problem of discrete system is studied. In addition, the improved PSO algorithm is also embodied in many aspects. Such as: binary PSO algorithm, w decrease with linear algebra evolution PSO algorithm, the parameter adaptive PSOalgorithm, and other intelligent algorithm with the combination of particle group and the mixed algorithm, and so on. In specific applications, PSO is widely used in neural network training, all kinds of parameter optimization of continuous and discrete problems (in the design of fuzzy controller, the robot path planning, signal processing and pattern recognition has achieved a good effect on issues), is applied to combinatorial optimization problems. In addition to the above areas, PSO in multi-objective optimization, automatic target detection, biological signal identification, decision-making scheduling, system identification and training of game also has obtained certain achievement. Although it has developed many kinds of 
different modified PSO algorithm, the performance also improves unceasingly, but, as a kind of optimization algorithm, particle swarm algorithm itself there are still many difficult problems, such as premature convergence, the selection of control parameters, etc. The existence of these problems, to practical application of particle swarm algorithm has brought great inconvenience. The performance of these algorithms are still remains to be further improved to better meet the actual requirements ${ }^{[4-7]}$.

\section{Related Works}

\subsection{Basic Ideas of Particle Group Method}

With similar genetic algorithm, PSO algorithm is a kind of based on iterative optimization tool, the system is initialized to a group of random solutions, through the iterative search for the optimal solution. But not particle swarm algorithm and genetic algorithm is adopted by the crossover and mutation operation. Compared with genetic algorithm, PSO algorithm has the advantage of the parameters of the need to adjust the small, simple structure, fast convergence speed. The particle swarm algorithm thought with simple, easy to implement, and application effect is obvious advantages and accepted by many application areas, and the adaptive control, combinatorial optimization, pattern recognition, machine learning, artificial life, management, decision-making, and other fields has been widely used. PSOalgorithm with strong robustness, especially to some large, complex optimization of nonlinear system, more show its unique and superior performance. PSO algorithm as an emerging subject, all kinds of theory, the method is not yet mature, needs further development and perfection. While in the process of research and application of particle swarm algorithm can appear many problems, at the same time also can produce many different kinds of algorithm design point of view, however, at present on the practical application of particle swarm algorithm has showed its excellent performance and huge development potential, and it is motivated by the prospect of development of all kinds of professional and technical personnel and the theory of the PSO method is applied to the practice of one's own work.

PSO algorithm is based on the evolution of the group. Reynolds of bird flight, according to a study bird just track it a limited number of neighbors, but the overall result is that the entire flock like in a central control, namely the global behavior of the complex is caused by the interaction of simple rules. PSO is the result of the study of birds feed on behavior, a flock of birds in the random search for food, if only a piece of food in this area, so the most simple and effective strategy is to find food and search for the nearest food around the area of the birds. PSO is get enlightenment from this model, and is used to solve optimization problem. In addition people are usually based on their own and others' experience as a basis for the decision-making, which constitutes the basic concept of PSO.

Algorithm using speed - position search model, and each particle represents a candidate solution of the solution space, the superiority of solution is determined by the fitness function. Speed of $V_{i}=\left(V_{i 1}, V_{i 2} \cdots V_{i d}\right)$ particles in search space displacement iteration. Among them, the fitness function based on the optimization goal definition. Particle swarm algorithm randomly initialized to a group of particles, in which the case of a particle in d I said the location of the solution space of $X_{i}=\left(X_{i 1}, X_{i 2} \cdots X_{i d}\right)$ compared with evolutionary algorithm, particle swarm algorithm retains the global search strategy based on population, but the pace of its USES a location model, the operation is simple, avoids the complex genetic operations. Its special memory that it can dynamically track the current the entire population of the optimal particle.

PSO algorithm in the optimization problem, the problem of the solution corresponding to the location of the search space of a bird, called the birds "particles" or "subject". They all have their own position and velocity (decided to the direction and distance of flight), 
there is a decided by be optimized function of adaptive value. Each particle memory, to follow the current optimum particles, search in the solution space.Each iteration of the process is not completely random, if find a good solution, will be on this basis to find a solution. The PSO is a group of random particles (random solutions), in each iteration, the particles by tracking two extremum 0 to update themselves: the first is the particle itself to find the best solution, called individual extreme value point, the global version of PSO another extreme value point is that the entire population now to find the best solution, called global extreme value point. Local version of PSO, the individual is to use some of the neighboring particles as a neighbor, all neighbors is the best solution in the local extreme value point[5-6].

\subsection{Operation Process and Application}

Particle swarm algorithm the main operation process description is as follows:

1) Population initialization. Randomly generated $M$ individuals as the initial group $Q(0)$. Due to the group as the operands of PSO, so we must for particle group operating an initial group made up of a number of initial solution.

2) Individual evaluation (fitness). Calculating the fitness of the individuals in groups.PSOalgorithm in the search process generally do not need other external information, only with the evaluation function value to evaluate the merits of the individual or solution, and as the basis of particle group after operation. Value is known as the fitness evaluation function.

3) Update the speed of particle group and location. This is the most crucial step in the whole PSO algorithm, the population of "individual learning" and "learning" collective are realized in this step.

4) Termination condition judgment. If meet the termination conditions (maximum number of iterations or satisfy the standard of minimum error), are given in the evolutionary process has the largest fitness of individuals as the optimal solution of the output, termination of calculation; Otherwise, turn to the first step, and continue to iteration. Particle swarm optimization algorithm flow chart is shown below: 


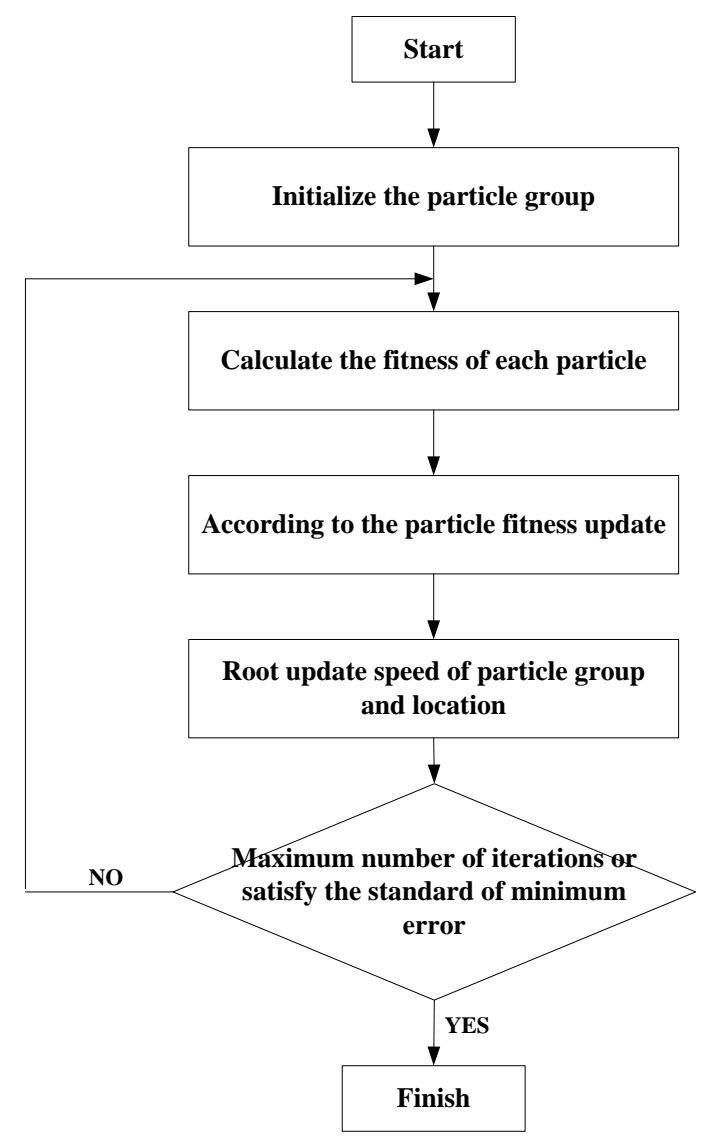

Figure 1. Particle Swarm Optimization Process

PSO algorithm has strong robustness, compared with traditional optimization technology, it has adopted many unique methods and techniques.

1) The traditional optimization algorithm is from an initial starting point, and then gradually iteration for the optimal solution. PSO is not, it is a group, at the same time more satisfied solution can be got with continual iteration.

2) The traditional optimization algorithm not only need to use the objective function value, and often require some derivative value of the objective function, and other auxiliary information to determine the search direction. Particle swarm algorithm to use only by the objective function value transformation of fitness function value can determine the search direction of further and search scope, without some derivative value of the objective function, and other auxiliary information.

3) The traditional optimization algorithms are mostly using deterministic search method, a point to another point search transfer to determine transfer relations and transfer direction, this kind of uncertainty often makes the search may never reach the most advantages, which limit the application range of the algorithm. And PSO is a group search method, has the potential adaptability.

Particle swarm optimization algorithm is based on the theory of swarm intelligence optimization algorithm, through the cooperation and competition between groups of particles produced by guidance of swarm intelligence optimization search. Compared with general evolutionary algorithm, particle swarm optimization algorithm is a more efficient parallel search algorithm, the concept is simple, easy to implement, generally only the short several lines of code, the advantage is obvious. 
PSO algorithm is a kind of evolutionary algorithm, but it did not use genetic operators to update the chromosome genes, but similar to the gradient descent algorithm makes the chromosome to conform to the highest degree function value of swimming. PSO algorithm was not able to give strict mathematical proof, but proved to be effective in practical application. The particle swarm algorithm has many advantages, but also exist algorithm accuracy is low, prone to spread such as faults, especially when this algorithm in the solution space search, can appear sometimes the phenomenon of oscillation near global optimal solution. Specific displays in: first, because the whole particle group are based on all the particles and their own search experience "flight", in the direction of the optimal solution under the effect of large acceleration coefficient, the particle group is likely to miss the optimum solution, away from the optimal solution of the divergence in the space, make the algorithm cannot convergence; Secondly, in the case of algorithm convergence, because all the particles to the optimal solution in the direction of the group of swimming, so all the particles tend to the same, lose the diversity between the particles, resulting in slow convergence speed in the late, at the same time the algorithm convergence to a certain accuracy, algorithm cannot continue optimization, algorithm can achieve the precision is $\operatorname{low}^{[7-10]}$.

\subsection{Mixing PSO Model}

PSO algorithm has strong robustness, compared with traditional optimization technology, it has adopted many unique methods and techniques.

1) The traditional optimization algorithm is from an initial starting point, and then gradually iteration for the optimal solution. PSO is not, it is a group, at the same time more satisfied solution can be got with continual iteration.

2) The traditional optimization algorithm not only need to use the objective function value, and often require some derivative value of the objective function, and other auxiliary information to determine the search direction. Particle swarm algorithm to use only by the objective function value transformation of fitness function value can determine the search direction of further and search scope, without some derivative value of the objective function, and other auxiliary information.

3) The traditional optimization algorithms are mostly using deterministic search method, a point to another point search transfer to determine transfer relations and transfer direction, this kind of uncertainty often makes the search may never reach the most advantages, which limit the application range of the algorithm. And PSO is a group search method, has the potential adaptability.

Particle swarm optimization algorithm is based on the theory of swarm intelligence optimization algorithm, through the cooperation and competition between groups of particles produced by guidance of swarm intelligence optimization search. Compared with general evolutionary algorithm, particle swarm optimization algorithm is a more efficient parallel search algorithm, the concept is simple, easy to implement, generally only the short several lines of code, the advantage is obvious.

PSO algorithm is a kind of evolutionary algorithm, but it did not use genetic operators to update the chromosome genes, but similar to the gradient descent algorithm makes the chromosome to conform to the highest degree function value of swimming. PSO algorithm was not able to give strict mathematical proof, but proved to be effective in practical application. The particle swarm algorithm has many advantages, but also exist algorithm accuracy is low, prone to spread such as faults, especially when this algorithm in the solution space search, can appear sometimes the phenomenon of oscillation near global optimal solution. Specific displays in: first, because the whole particle group are based on all the particles and their own search experience "flight", in the direction of the optimal solution under the effect of large acceleration coefficient, the particle group is likely to miss 
the optimum solution, away from the optimal solution of the divergence in the space, make the algorithm cannot convergence; Secondly, in the case of algorithm convergence, because all the particles to the optimal solution in the direction of the group of swimming, so all the particles tend to the same, lose the diversity between the particles, resulting in slow convergence speed in the late, at the same time the algorithm convergence to a certain accuracy, algorithm cannot continue optimization, algorithm can achieve the precision is low $^{[11-13]}$.

$$
\begin{gathered}
\operatorname{Child}_{1}\left(Y_{i}\right)=P_{i} \times \operatorname{parent}_{1}\left(Y_{i}\right)+\left(1-P_{i}\right) \times \operatorname{parent}_{1}\left(Y_{i}\right) \\
\operatorname{Child}_{2}\left(Y_{i}\right)=P_{i} \times \operatorname{parent}_{2}\left(Y_{i}\right)+\left(1-P_{i}\right) \times \operatorname{parent}_{2}\left(Y_{i}\right) \\
\operatorname{Child}_{1}\left(X_{i}\right)=\frac{\operatorname{parent}_{1}\left(Y_{i}\right)+\operatorname{parent}_{2}\left(Y_{i}\right)}{\left|\operatorname{parent}_{1}\left(Y_{i}\right)+\operatorname{parent}_{2}\left(Y_{i}\right)\right|}\left|\operatorname{arent}_{1}\left(Y_{i}\right)\right| \\
\operatorname{Child}_{2}\left(X_{i}\right)=\frac{\operatorname{parent}_{1}\left(Y_{i}\right)+\operatorname{parent}_{2}\left(Y_{i}\right)}{\left|\operatorname{parent}_{1}\left(Y_{i}\right)+\operatorname{parent}_{2}\left(Y_{i}\right)\right|}\left|\operatorname{parent}_{2}\left(Y_{i}\right)\right|
\end{gathered}
$$

Type of PSO and the only difference is that the traditional PSO model particle group in speed and position is updated to the crossover operation, and produce grain particles to replace the offspring of the parents. Crossover operation make the offspring inherited the merits of the parent particles particles, in theory, to strengthen the area between the particles search ability. For example, both parents particles in a different area of the local optimum, so both cross offspring produced by the particles tend to get rid of local optimum, and improved the search results. Experiments show that compared with the traditional PSO and traditional genetic algorithm, crossover PSO search speed, convergence precision is high. At present, the traditional PSO algorithm was modified using evolutionary operation exploration continues.

\section{Mixing of PSO in the Application of the Network Routing Optimization}

After entering the information age, people living in the modern society and all kinds of network is closely linked. By the computer information network, telephone communication network, transport, energy and logistics service network dispatch network composed of various network such as network group of penetrated into every aspect of life. The purpose of network optimization is to research how to effectively plan, manage, and control the network system, to maximize social benefit and economic benefit. Network optimization is a classical and important branch of operational research, the study of problems involved in many fields, on the one hand is how to maximize resource conservation, such as the shortest path problem, minimum cost problem; On the other hand, in the case of limited network resources how to play the biggest benefit, such as the maximum flow problem, the optimal resource allocation problem, and so on.

Routing information through the Internet channel from the source to the destination. Along the route, the information by at least an intermediate point, how to choose by the number of basis points, to meet the requirements of information transmission is very concern of the network users. Can choose different information transmission requirements and adopt different routing method. Routing method is first to determine a standard to measure, such as the length of the path, bandwidth or path on the basis of the number, etc., to determine the best route to reach the objectives, and then from the corresponding methods in the routing table to select an appropriate routing, will be sent packet along the chosen path.

Routing algorithm USES many different metrics to determine the best path. Complex 
routing algorithm can choose routing based on multiple measures, and combine them into a composite metric. The commonly used measurement is as follows:(1) The path length, (2) The bandwidth, (3) The reliability, (4) The communication overhead, (5) Load, (6) Time delay

Routing algorithm of tasks are correctly transfer business flow from the source destination basis points, to meet the business quality of service, at the same time maximize the performance of the network and minimize transmission cost, network limited ability of exchange and transmission is additional constraints. One of the routing optimization goal is to improve the network performance, network performance index is usually the throughput and average delay. Routing is the indispensable function in a variety of communication network, it according to the distribution of network business flow, for each call reasonably arrange routing, ensure the efficient and reliable transmission of information, make the network produces the biggest economic benefits.

In order to provide high performance and high quality service, the next generation Internet is facing great challenge, must be able to provide guarantee service quality and bandwidth. Service quality is IP network in accordance with the requirements of the application of successful transmission of IP packets. The current Pl only supports the "best" solution, just try your best to send the packet network, there is no guarantee of packet loss and delay index; In the case of resources fully can provide good service quality, but in the case of limited resources service quality is poor. As a result, the end-to-end IP service quality is the key of the research. IP quality of service description IP data flow through the network's performance features, this feature can be expressed by the collection of the following parameters: the reliability of the transmission service, the reliability of the network connection to the end through your fingers; Time delay which is also called the time lag, refers to the transmission delay between two reference points; Delay jitter, refers to the same routing data flow through the same when measured interval of delay difference; According to in the network transmission rate, throughput, index average rate or peak rate expression is available; Packet loss rate, refers to the biggest ratio data loss during transmission.

Quality of service routing can be classified into two categories: unicast routing, and multicast routing. Unicast routing problem are defined as follows: given a source point a, the objective basis points $b$, a collection of service quality restriction $G$ and possible optimization index, found that meet the needs of $\mathrm{C}$ the best feasible path from a to $\mathrm{b}$. Unicast routing problem contains four basic quality of service routing problem: link constraint problem, link optimization problem, the path constraint

And the path optimization problem. Multicast routing problem are defined as follows: given a source point a, the objective basis points set $\mathrm{R}$, a collection of service quality restriction $G$ and possible optimization index, find meet $G$ and traverse $a$ and $R$ all points of the optimal routing tree. Multicast routing problem is similar to unicast routing process. The difference only lies in the fact that the optimization of path in unicast routing or constraint, to right in the multicast routing by the operation of the tree. For example, bandwidth optimization routing is looking for the biggest bottleneck bandwidth routing tree; Is time delay constrained routing routing tree, from the source to any objective basis points of time delay is less than the given value.

Mixed particle swarm algorithm to solve the service quality of unicast routing problem, steps can be divided into the following steps:

1) Initialize the network basis: the value $\left(d_{i}, d j_{i}, p l_{i}\right)$ basis points, and each of the boundary value of $\left(d_{i j}, d j_{i j}, b_{i j}, c_{i j}\right)$ constraint conditions are given in the value of $\mathrm{D}, \mathrm{DJ}, \mathrm{B}, \mathrm{PL}$. For the probability of each edge (i,j) set an initial value, and the initialization $\mathrm{m}$ particles;

2) According to the weight of each basis point cut some does not satisfy the constraint conditions. Basis points, linked to the basis points should also be deleted;

3) According to the weight of each edge of the cut some does not meet the constraint conditions of edge

4) According to the mixed strategy of particle swarm algorithm for the corresponding 
operation, continuous iteration, eventually find a satisfy all constraints routing[14-15].

\section{The Simulation Results}

Consider a small network, as shown in figure 2, carries on the simulation. Assume that three unicast routing requests $(1,7),(2,6)$ and $(3,8)$. They are the requirements for the quality of service: $\mathrm{B}=70, \mathrm{D}=8, \mathrm{DJ}=5, \mathrm{PL}=0.0001$. Blending algorithm of parameter selection is: because the network of the relatively small size, so the number of particles for the 10 would be sufficient, evolution algebra was $\$ 100$, still the idea of mixing of PSO algorithm, by mutation and crossover operation of genetic algorithm to improve the basic PSO algorithm. Combining with solving problem with constraint condition in front of the train of thought, generate enough particles effectively, make the problem can be easily solved.

Figure 2 is the network of the original figure, shows the various points of the model and topology structure of the attachment of the edge and position.

As shown in figure 3 , according to the constraint conditions of network bandwidth $(e) \geq B$, solving the model. Because of basis points between 2 and 3 , the bandwidth of the basis points between 4 and 8 do not meet the conditions, so they are removed.

Based on package is shown in figure 4 presented.according to the constraints of the packet_loss $(n) \leq P L$, solving the model.

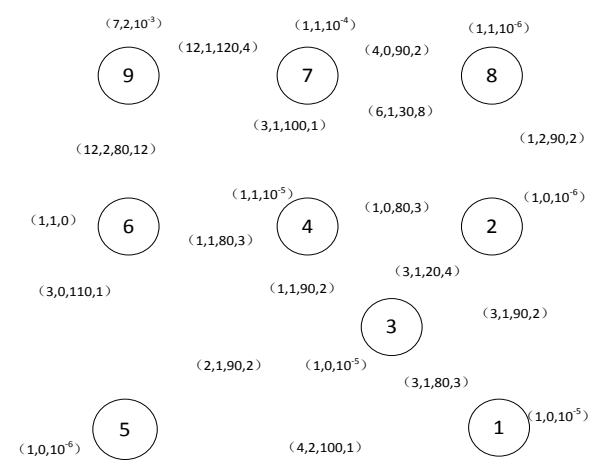

Figure 2. Network Original Figure

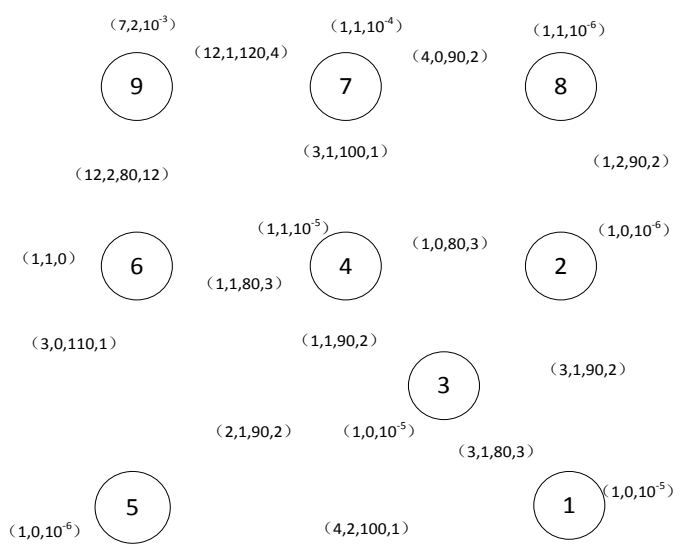

Figure 3. Diagram of the Application with Loose Constraint Conditions 


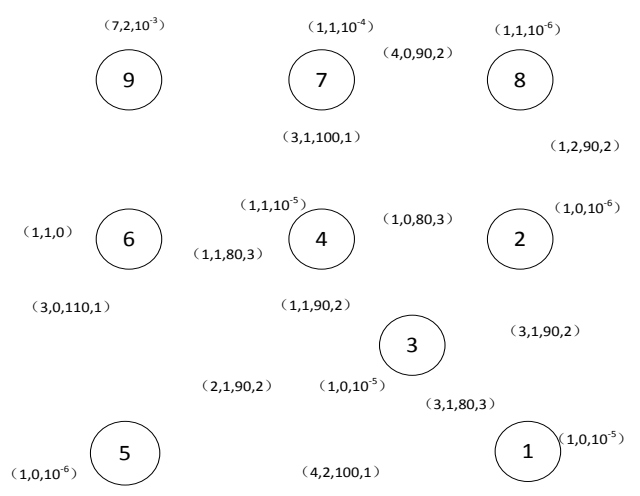

\section{Figure 4. Diagram of the Application Package Presented. According to the Constraint}

Global Optimization result is obtained by simulation as shown in table 1 .

Table 1. The Simulation Results

\begin{tabular}{|c|c|c|c|c|}
\hline \multirow{2}{*}{ Routing requests } & \multicolumn{4}{|c|}{ QoS routing results } \\
\cline { 2 - 5 } & Select the routing & Cost & Time delay & Delay jitter \\
\hline$(1,7)$ & $1-2-4-6-7$ & 9 & 11 & 4 \\
\hline$(2,6)$ & $2-4-6$ & 6 & 3 & 1 \\
\hline$(3,8)$ & $3-4-2-8$ & 7 & 5 & 3 \\
\hline
\end{tabular}

\section{Conclusion}

Network routing optimization problem is similar to the TSP problem, belong to the combinatorial optimization of discrete problem; The difference is the TSP problem is to find meet closed loop with the shortest total distance and network routing optimization is to find satisfying the constraint condition of basis points from the source to the purpose of a optimal path. This chapter introduces the network routing optimization, on the basis of relevant knowledge, will be mixed PSO algorithm is used in the IP network routing optimization. First discussed the basic routing algorithm and routing protocol; Then mixed particle swarm algorithm is illustrated by examples in the application of network routing optimization; Finally analyzed the mixed particle swarm algorithm based IP network service quality unicast routing. Specific algorithm model is put forward, through the numerical example to verify the effectiveness of the proposed algorithm can well solve the problem of IP network service quality unicast routing.

\section{Reference}

[1] J. Kennedy, "Particle swarm optimization[M]//Encyclopedia of Machine Learning", Springer US, (2010), pp.760-766.

[2] R. Poli, J. Kennedy and T. Blackwell, "Particle swarm optimization", Swarm intelligence, vol.1, no.1, (2007), pp.33-57.

[3] R. Poli, J. Kennedy and T. Blackwell, "Particle swarm optimization", Swarm intelligence, vol.1, no.1, (2007), pp.33-57.

[4] G. Venter and J. Sobieszczanski-Sobieski, "Particle swarm optimization”, AIAA journal, vol.41, no.8, (2003), pp.1583-1589.

[5] R. C. Eberhart and Y. Shi, "Particle swarm optimization: developments, applications and resources[C]//Evolutionary Computation, 200", Proceedings of the 2001 Congress on. IEEE, (2001).

[6] R. C. Eberhart and J. Kennedy, "A new optimizer using particle swarm theory", Proceedings of the sixth 
international symposium on micro machine and human science, (1995).

[7] R. C. Eberhart and Y. Shi, "Comparing inertia weights and constriction factors in particle swarm optimization[C]//Evolutionary Computation, 2000", Proceedings of the 2000 Congress on. IEEE, (2000).

[8] M. Caesar, M. Castro and E. B. Nightingale, "Virtual ring routing: network routing inspired by DHTs", ACM SIGCOMM Computer Communication Review. ACM, vol.36, no.4, (2006), pp.351-362.

[9] J. C. Cano and P. Manzoni, "A performance comparison of energy consumption for mobile ad hoc network routing protocols[C]//Modeling, Analysis and Simulation of Computer and Telecommunication Systems, 2000”, Proceedings. 8th International Symposium on. IEEE, (2000).

[10] J. C. Cano and P. Manzoni, "A performance comparison of energy consumption for mobile ad hoc network routing protocols[C]//Modeling, Analysis and Simulation of Computer and Telecommunication Systems, 2000. Proceedings”, 8th International Symposium on. IEEE, (2000).

[11] C. Ozturk, Y. Zhang and W. Trappe, Source-location privacy in energy-constrained sensor network routing[C]//SASN, vol.4, (2004), pp.88-93.

[12] N. Minar, K. H. Kramer and P. Maes, "Cooperating mobile agents for dynamic network routing[M]//Software agents for future communication systems", Springer Berlin Heidelberg, (1999), pp. 287-304.

[13] B. A. Carré, "An algebra for network routing problems", IMA Journal of Applied Mathematics, vol.7, no.3, (1971), pp.273-294.

[14] C. L. Barrett, S. J. Eidenbenz and L. Kroc, "Parametric probabilistic sensor network routing”, Proceedings of the 2nd ACM international conference on Wireless sensor networks and applications. ACM, (2003).

[15] C. L. Barrett, S. J. Eidenbenz and L. Kroc, "Parametric probabilistic sensor network routing", Proceedings of the 2nd ACM international conference on Wireless sensor networks and applications. ACM, ( 2003).
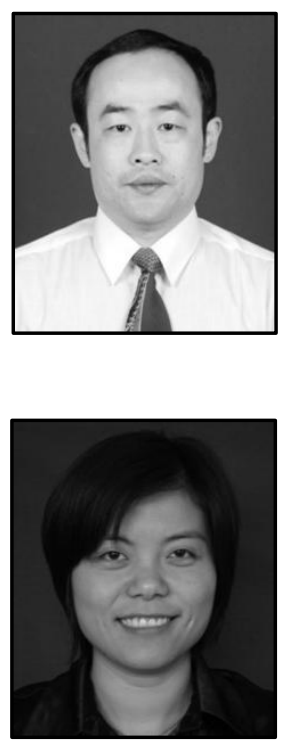

\section{Authors}

Sheng Yan Gang, he received the Bachelor's degree in Computer Application from Tianjin University of Technology and Education and the Master's degree in Computer Software and Theory from Shandong University, China in 2001 and 2010 respectively. He is a faculty member in Qingdao Vocational and Technical College of Hotel Management. He is currently researching on the technology of Computer network and Communication.

Tong Fei, she received the Bachelor's degree in Computer Science and Technology from Qingdao Technological University and the Master's degree in Computer Application from Ocean University of China, China in 2003 and 2009 respectively. She is faculty member in Qingdao Vocational and Technical College of Hotel Management. She is currently researching on the technology of Computer network and Communication. 
International Journal of Future Generation Communication and Networking Vol. 8, No. 4 (2015) 